\title{
BMJ Open Increasing incidence of recent hepatitis $C$ virus infection among persons seeking voluntary counselling and testing for HIV and sexually transmitted infections in Taiwan
}

\author{
Jen-Chih Tsai, ${ }^{1}$ Chien-Ching Hung, ${ }^{2}$ Sui-Yuan Chang, ${ }^{3,4}$ Wen-Chun Liu, ${ }^{2}$ \\ Cheng-Hsin Wu, ${ }^{2}$ Yi-Ching Su, ${ }^{2}$ Pei-Ying Wu, ${ }^{5}$ Yu-Zhen Luo, ${ }^{5}$ Lan-Hsin Chang, ${ }^{2}$ \\ Hsin-Yun Sun, ${ }^{2}$ Shan-Chwen Chang $^{2}$
}

To cite: Tsai J-C, Hung C-C, Chang S-Y, et al. Increasing incidence of recent hepatitis $C$ virus infection among persons seeking voluntary counselling and testing for HIV and sexually transmitted infections in Taiwan. BMJ Open 2015;5:e008406. doi:10.1136/bmjopen-2015008406

- Prepublication history and additional material is available. To view please visit the journal (http://dx.doi.org/ 10.1136/bmjopen-2015008406).

Received 5 April 2015

Revised 5 August 2015

Accepted 17 September 2015

CrossMark

For numbered affiliations see end of article.

Correspondence to

Dr Hsin-Yun Sun;

hysun13@gmail.com

\section{ABSTRACT}

Objectives: The incidence of hepatitis $\mathrm{C}$ virus (HCV) infection among HIV-negative men who have sex with men (MSM) is rarely investigated in the Asia-Pacific region. We aimed to estimate the incidence rate of and factors associated with recent HCV infection among the clients seeking voluntary counselling and testing (VCT) services for HIV in Taiwan.

Methods: During 2006-2013, 12143 clients sought VCT services for HIV. Clients with subsequent follow-up tests at an interval of 6 months or longer were included to estimate the incidence rate of HCV seroconversion. Phylogenetic analysis of HCV sequences from VCT clients and HIV-positive patients was performed.

Results: The overall HCV seroprevalence at baseline was $0.3 \%$. Of 2150 clients testing negative for anti-HCV antibody at baseline with a total of 5074.99 personyears of follow-up (PYFU), 17 (0.8\%) developed HCV seroconversion, leading to an overall incidence rate of 3.35 per 1000 PYFU $(95 \% \mathrm{Cl} 1.76$ to 4.94$)$, which increased from $2.28(95 \% \mathrm{Cl} 0.05$ to 4.51$)$ in 20062009, to 3.33 (95\% Cl 0.86 to 5.80) in 2010 to 2011 and 4.94 per 1000 PYFU $(95 \% \mathrm{Cl} 0.99$ to 8.99$)$ in 2012-2013; the incidence of early syphilis increased from 11.91 to 13.28 and 31.78 per 1000 PYFU in the three corresponding periods. In multivariate analysis, having HIV-positive partners (adjusted HR (AHR) $=3.756 ; 95 \% \mathrm{Cl} 1.180$ to 11.955$)$ and developing a rapid plasma reagin titre of 4 or greater $(\mathrm{AHR}=9.978 ; 95 \% \mathrm{Cl}$ 1.550 to 64.233 ) were significantly associated with HCV seroconversion.

Conclusions: An increasing trend of recent HCV infection occurs among individuals seeking VCT services in Taiwan. Having HIV-positive partners and having syphilis are independently associated with recent HCV seroconversion.

\section{INTRODUCTION}

After the introduction of combination antiretroviral therapy, AIDS-related morbidity

\section{Strengths and limitations of this study}

- This is the first study in the Asia-Pacific region to estimate hepatitis C virus (HCV) incidence among individuals seeking voluntary counselling and testing services, particularly HIV-negative men who have sex with men (MSM).

- This study demonstrates the increasing incidence of HCV infection in high-risk HIV-negative MSM and provides useful information to clinical physicians.

- Owing to the small number of cases and single hospital study, more surveillance studies are warranted.

and mortality have decreased significantly, and end-stage liver disease has become the leading cause of non-AIDS-related deaths in the HIV-positive population, especially among those co-infected with hepatitis $\mathrm{C}$ virus $(\mathrm{HCV}) .{ }^{1}$ While parenteral transmission remains the most efficient route of $\mathrm{HCV}$ infection, ${ }^{2}$ sexual contact has not been considered as an important route for HCV transmission. ${ }^{3}$ In the USA and Western Europe, $\mathrm{HCV}$ seroprevalence is 72-95\% among HIV-positive persons with a history of injection drug use, $1-12 \%$ in HIV-positive men who have sex with men (MSM) and 9-27\% in HIV-positive heterosexuals. ${ }^{4}$ In Taiwan, $\mathrm{HCV}$ seroprevalence is $96.8 \%$ among HIV-positive persons with a history of injection drug use, $5.5 \%$ in HIV-positive MSM and $10.9 \%$ in HIV-positive heterosexuals. ${ }^{5-8}$

Since 2000, outbreaks of acute HCV infection among HIV-positive MSM without a history of injection drug use have been increasingly reported, ${ }^{9-12}$ with incidence rates ranging from 1-3 per 1000 person-years 
(PY) to 10 or more per $1000 \mathrm{PY}^{13-18}$ Several factors have been identified to be associated with acquisition of HCV infection via sexual contacts among HIV-positive MSM, such as unprotected receptive anal intercourse with multiple partners and syphilis, rectal trauma with bleeding, fisting and use of non-parenteral recreational drugs. ${ }^{19-22}$

However, there are limited data about the incidence of HCV infection among HIV-negative MSM, especially in the Asia-Pacific region. In the UK, unselected screening of HCV among MSM over a 6-month period when they attended sexual health clinics showed similar HCV seroprevalence between the general UK population $(0.72 \%)$ and MSM with HIV-negative or unknown HIV status $(0.61 \%){ }^{23}$ However, in a study by Richardson et $a l,{ }^{15}$ the incidence of HCV infection in HIV-negative MSM increased from 0 per 1000 PY in 2000-2003 to 5.8 per $1000 \mathrm{PY}$ in 2006.

Our previous study in Taiwan has shown an increasing trend of recent HCV infection among HIV-positive MSM between 2000 and 2010, which was associated with syphilis. ${ }^{18}$ In this study, we aimed to estimate the incidence rate of recent HCV infection among individuals who were not injecting drug users (IDUs), and who sought voluntary counselling and testing (VCT) services for HIV and sexually transmitted infections (STIs) at a university hospital in Taiwan. Preliminary results of this study were presented in the 2014 HIV Drug Therapy Glasgow Congress. ${ }^{24}$

\section{METHODS}

Study setting

VCT services for HIV and syphilis were offered free of charge in Taiwan, with grant support from Taiwan Centers for Disease Control. ${ }^{25}$ Every client seeking VCT services would have a unique code for identification of test results, and complete an anonymous, selfadministered questionnaire to obtain information on the demographics, sexual practices, risk behaviours for HIV infection, prior or current STIs, number of sexual partners, HIV serostatus of sexual partners, condom use and use of injection drugs or non-injecting recreational drugs. After completion of integrated pretesting and post-testing counselling, a blood sample was obtained for testing for HIV, syphilis and viral hepatitis. ${ }^{25}$

In this study, all clients who sought VCT services between May 2006 and December 2013 were included, and clients using the same code who sought VCT services more than two times with an interval of 6 months or longer were eligible for inclusion to estimate the incidence rate of $\mathrm{HCV}$ seroconversion. IDUs and clients who sought VCT services only once or with repeat tests performed at an interval of less than 6 months were excluded. The participants gave written informed consent by using the unique code.

\section{Laboratory investigations}

Antibodies to $\mathrm{HCV}$ were determined with a fourthgeneration enzyme immunoassay (GB NANBASE C-96,
V4.0). The HCV NS5B gene sequences, amplified from VCT clients with positive HCV antibody and patients co-infected with HIV and HCV in our cohort, between 2006 and 2013, were used in phylogenetic analysis. A 336-bp fragment covering partial HCV NS5B (nucleotides (nt) 8294-8629 relative to HCV reference strain H77) was amplified by PCR as previously described. ${ }^{18}$ The final PCR products were sequenced. Phylogenetic analysis was performed to determine the HCV genotypes by the use of NS5B sequences amplified from HCV-positive patients in our cohort. ${ }^{18} 26$ The study and reference sequences were aligned using the Clustal W program with minor manual adjustment. The tree was constructed by the neighbour-joining method based on the Kimura two-parameter distance matrix listed in MEGA software (V.3.0). ${ }^{27}$

\section{Nucleotide sequence accession numbers}

The GenBank accession numbers of the obtained partial NS5B HCV sequences used in the phylogenetic analysis were JQ060117-121, JQ060123-124, JQ060126, JQ060131-133, HM031199, HM031203-204, HM031211, DQ179117，DQ663604，DQ663608，DQ666268 and EU255966. The GenBank accession numbers for the four seroconversion sequences were 1847251, 1847258, and 1847261-62.

\section{Definitions}

Recent HCV seroconversion was defined as the first positive HCV antibody that was detected within 1 year after the previous negative HCV antibody. The date of seroconversion was arbitrarily assigned as the midpoint between the date of the last negative HCV antibody and that of the first positive HCV antibody. ${ }^{18}$ Recent syphilis was defined as having an rapid plasma reagin (RPR) titre with a fourfold increase or new RPR seroreactivity within 6 months of $\mathrm{HCV}$ seroconversion or within 6 months of the last anti-HCV test. $^{18}$

\section{Statistical analyses}

All statistical analyses were performed by SPSS software, V.17 (SPSS Inc, Chicago, Illinois, USA). Categorical variables were compared using $\chi^{2}$ or Fisher's exact test whereas non-categorical variables were compared using Student's t test or Mann-Whitney U test. All tests were two-tailed and a $\mathrm{p}$ value $<0.05$ was considered significant. Cox proportional hazards model was used to identify factors associated with HCV seroconversion. All included clients were followed from the date of first VCT visit during the study period to the date of the last VCT visit or HCV conversion. To assess the trend of HCV infection, the follow-up duration was further categorised into three time periods, 2006-2009, 2010-2011 and 2012-2013. The incidence rate of HCV seroconversion in each study period was calculated as number of episodes of HCV seroconversion per $1000 \mathrm{PY}$ of follow-up (PYFU). Poisson regression was used for comparisons of incidence rates in three time periods (2006-2009, 2010-2011 and 
2012-2013). A nested case-control study was also performed to compare the clinical characteristics of $\mathrm{HCV}$ seroconverters with those of HCV non-seroconverters. Every HCV seroconverter was matched with four nonseroconverters for age, gender, year of first VCT visit and duration of follow-up during the study period.

\section{RESULTS}

Between May 2006 and December 2013, 12143 individuals sought VCT services and underwent a total of 18246 tests, and 9986 clients were excluded in the further analysis because of being IDUs or having only one test during the period. The remaining 2157 clients contributed a total of 8260 tests with an interval of 6 months or longer between two tests.

At baseline, HCV seropositivity was found in seven clients $(0.3 \%)$. Of the 2150 clients with negative HCV antibody at baseline who contributed a total of 5074.99 PYFU, $17(0.8 \%)$ had HCV seroconverted during the follow-up. This led to an overall incidence rate of 3.35 per 1000 PYFU (95\% CI 1.76 to 4.94$)$. The clinical characteristics of the $17 \mathrm{HCV}$ seroconverters and 2133 clients who remained seronegative for HCV are shown in table 1 . Thirteen of the $17(76.5 \%) \mathrm{HCV}$ seroconverters were MSM and $4(23.5 \%)$ were heterosexuals.

During the three study periods, the incidence rate of HCV seroconversion increased from 2.28 per 1000 PYFU (95\% CI 0.05 to 4.51 ) in 2006-2009 and 3.33 per 1000 PYFU (95\% CI 0.86 to 5.80) in 2010-2011, to 4.94 per 1000 PYFU (95\% CI 0.99 to 8.99) in 2012-2013 (figure 1). The incidence rate of syphilis also increased from 11.91 per 1000 PYFU in 2006-2009 to 31.78 per 1000 PYFU in 2012-2013 (figure 1); the rates were significantly higher in HCV seroconverters than non-seroconverters during the three corresponding study periods.

In multivariate analysis, HCV seroconverters were more likely to have HIV-positive partners (adjusted HR (AHR) 3.756; 95\% CI 1.180 to 11.955 ) and a RPR titre of four or greater (AHR 9.978; 95\% CI 1.550 to 64.233) than HCV non-seroconverters (table 2). In the nested case-control study, the proportion of clients having HIV-positive sexual partners remained higher in the HCV seroconverters than in the non-seroconverters (23.5\% vs $3.1 \%, \mathrm{p}=0.016$ ), so did the proportion of those who had recent syphilis $(11.8 \%$ vs $0 \%, \mathrm{p}=0.04)$ and an RPR titre of four or greater $(11.8 \%$ vs $0 \%$, $\mathrm{p}=0.04$ ). During follow-up, HCV seroconverters tended to be more likely to become HIV positive than nonseroconverters $(17.7 \%$ vs $3.1 \%, \mathrm{p}=0.059)$ (see online supplementary table S1). In multivariate analysis, having HIV-positive sexual partners remained independently associated with HCV seroconversion with an adjusted OR of 6.931 (95\% CI 1.064 to 45.163 ) (see online supplementary table S2).

Among the $17 \mathrm{HCV}$ seroconverters, four clients had HCV viraemia detected by the PCR assay. One client was infected with $\mathrm{HCV}$ genotype 2a, and three with genotype 1b. Phylogenetic analysis was performed to determine their relationship with prevalent HCV sequences of HIV-positive MSM $(n=11)$ and IDUs $(n=9)$. The sequence of HCV isolates from HCV seroconverts among the VCT clients appeared to cluster with those from HIV-positive MSM, rather than those of IDUs (figure 2).

\section{DISCUSSION}

In this study, we demonstrate an increasing trend of HCV seroconversion among VCT clients who were not

Table 1 Clinical characteristics of HCV seroconverters and non-seroconverters

\begin{tabular}{|c|c|c|c|}
\hline Characteristics & $\begin{array}{l}\text { Seroconverters } \\
N=17(\%)\end{array}$ & $\begin{array}{l}\text { Non-seroconverters } \\
\mathrm{N}=2133(\%)\end{array}$ & p Value \\
\hline Male, n (\%) & $17(100.0)$ & $1988(93.2)$ & 0.6236 \\
\hline Age $($ mean $\pm S D)$, years & $28.0( \pm 6.21)$ & $29.0( \pm 6.5)$ & 0.5109 \\
\hline \multicolumn{4}{|l|}{ Risk, $n(\%)$} \\
\hline MSM & $13(76.5)$ & $1545(72.4)$ & \multirow[t]{2}{*}{0.766} \\
\hline Heterosexual & $4(23.5)$ & $587(27.5)$ & \\
\hline \multicolumn{4}{|l|}{ Reasons for screening, $\mathrm{n}(\%)$} \\
\hline HIV-positive sexual partners & $4(23.5)$ & $195(9.1)$ & 0.0649 \\
\hline History of STI & $2(11.8)$ & $318(14.9)$ & 0.7168 \\
\hline Having sex-for-money or money-for-sex & $2(11.8)$ & $153(7.2)$ & 0.3498 \\
\hline Having one-night stands & $4(23.5)$ & $568(26.6)$ & 0.7733 \\
\hline Having anal sex & $11(64.7)$ & 1109 (52.3) & 0.3081 \\
\hline Having unsafe anal sex (without $100 \%$ condom use) & 5 (33.3) & $531(25.4)$ & 0.7329 \\
\hline Having oral sex & $12(70.6)$ & $1444(68.1)$ & 0.823 \\
\hline Recreational drug use & $2(11.8)$ & $148(6.9)$ & 0.4366 \\
\hline \multicolumn{4}{|l|}{ Laboratory test results, $\mathrm{n}(\%)$} \\
\hline HIV seroconversion during follow-up & $3(17.7)$ & $132(6.2)$ & 0.0863 \\
\hline RPR titre of $\geq 4$ & $2(11.8)$ & $41(1.9)$ & 0.0439 \\
\hline Recent syphilis & $2(11.8)$ & $82(3.8)$ & 0.1406 \\
\hline
\end{tabular}




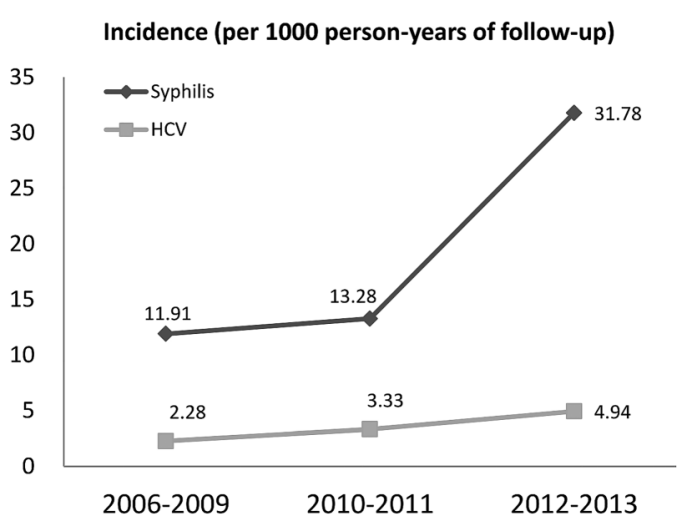

Figure 1 The incidence rates of hepatitis $\mathrm{C}$ virus $(\mathrm{HCV})$ and syphilis, 2006-2013.

IDUs, which increased from 2.28 per 1000 PYFU in 2006-2009 to 4.94 per 1000 PYFU in 2012-2013. Having HIV-positive partners was a consistently independent factor associated with HCV seroconversion in two multivariate analyses. Furthermore, the phylogenetic analysis corroborated these results and showed that sequences from HCV seroconverters among VCT clients tended to cluster with those from HIV-positive MSM.

Similar to the findings observed in several Western countries, the increasing trends of $\mathrm{HCV}$ infection in HIV-positive MSM have also been found in East Asia, in regions of Japan, ${ }^{28}$ Hong Kong $^{29}$ and Taiwan, ${ }^{18}$ in recent years. However, few studies addressed the incidence rates of HCV infection for transmission of STIs among the HIV-negative populations who engaged in high-risk behaviours. In the van de Laar et al ${ }^{13}$ study, there were no HCV seroconverters among HIV-negative MSM during two study periods (1984-1999 and 20002003). In contrast, Richardson et $a l^{15}$ reported that the incidence of HCV infection in HIV-negative MSM increased from 0 per 1000 PY during 2000-2003, 0.7 per $1000 \mathrm{PY}$ in 2004 and 1.5 per $1000 \mathrm{PY}$ in 2005 , to 5.8 per $1000 \mathrm{PY}$ in 2006. Such discrepancies might result from a lack of data from HIV-negative MSM in the former study because regular HCV screening was not recommended for MSM without HIV infection when the study was conducted. In our study, the increasing incidence of HCV infection in HIV-negative MSM concurs with the findings of Richardson et al. Such findings would support the counselling at VCT visit that provides information, communication and education about traditional STIs, such as syphilis, gonorrhoea and chlamydia, as well as viral hepatitis.

In the two analyses, we found that having HIV-positive partners was consistently an independent factor associated with HCV seroconversion, while other risk factors, such as history of STIs, anal sex, one-night stands, unsafe anal sex (defined as no $100 \%$ condom use), oral sex and use of non-injection drugs, were not statistically significantly different between HCV seroconverters and non-seroconverters. HIV infection may increase both infectiousness and susceptibility to HCV. In the study by Sherman et al, ${ }^{30}$ HIV increased serum HCV RNA load by more than $1 \log _{10} \mathrm{IU} / \mathrm{mL}$ in HCV/HIV-co-infected individuals compared to those with $\mathrm{HCV}$-mono-infection, and HCV/HIV-co-infected patients were more likely to shed HCV RNA in semen compared with HCV-mono-infected patients, suggesting a possible role in sexual transmission. ${ }^{31}$ Although we did not have HCV data from those HIV-positive partners, the finding of clustering of HCV sequences in phylogenetic analysis between HIV-positive and HIV-negative MSM in our study suggests such a link of HCV transmission.

Our study found that an RPR titre of four or greater was associated with HCV seroconversion in multivariate analysis (table 2). Ulcerative STIs, such as syphilis or lymphogranuloma venereum (LGV), could facilitate HCV acquisition from disrupted mucosa. In a case-control study, ${ }^{22}$ individuals with a history of syphilis, gonorrhoea or chlamydia in the past 12 months were associated with HCV seroconversion in univariate analysis (OR 2.78, $95 \%$ CI 1.18 to 6.5$)$. In the study by Sun $e t a l,{ }^{18}$ in Taiwan, HIV-positive MSM with recent syphilis had an approximately 7.3-fold higher risk of HCV seroconversion than those without recent syphilis. Our finding of concurrently increasing trends of syphilis lends support to the association between syphilis and HCV seroconversion among our VCT clients (figure 1).

Changes of risk behaviours may also be an important issue in sexual transmission of HCV infection. In London, a large survey of changes in sexual behaviours of MSM was performed between 1998 and 2008. ${ }^{32}$ The percentage of MSM who had unprotected anal intercourse (UAI) increased from $24.3 \%$ in 1998 to $36.6 \%$ in 2008 . In another UK study, ${ }^{33} 60$ cases and 130 controls showed that UAI, rimming, fisting, use of sex toys and group sex

Table 2 Multivariate analysis of factors associated with HCV seroconversion among all clients

\begin{tabular}{|c|c|c|c|}
\hline Variables & Reference & AHR (95\% Cl) & p Value \\
\hline Age, years & Continuous variable & $0.927(0.843$ to 1.02$)$ & 0.1209 \\
\hline HIV-positive sexual partners & Unknown or HIV-negative sexual partners & 3.756 (1.18 to 11.955$)$ & 0.0251 \\
\hline MSM & Heterosexual & $0.852(0.267$ to 2.761$)$ & 0.7867 \\
\hline Use of recreational drugs & No use of recreational drugs & $1.252(0.241$ to 6.5$)$ & 0.7889 \\
\hline History of STI & No history of STI & $0.332(0.061$ to 1.811$)$ & 0.2031 \\
\hline RPR titre of $\geq 4$ & RPR titre of $<4$ & 9.978 (1.55 to 64.233$)$ & 0.0155 \\
\hline
\end{tabular}


Figure 2 Phylogenetic analysis of NS5B sequences amplified from HCV strains from HCV seroconverters (closed triangles), HIV-positive MSM (closed circles) and HIV-positive injecting drug users (open squares). The GenBank accession numbers of the obtained partial NS5B HCV phylogenetic analysis were JQ060117-121, JQ060123-124, JQ060126, JQ060131-133, HM031199, HM031203-204, HM031211, DQ179117, DQ663604, DQ663608, DQ666268 and EU255966 (HCV, hepatitis $C$ virus; IDU, injecting drug user; MSM, men who have sex with men; VCT, voluntary counselling and testing). sequences used in the

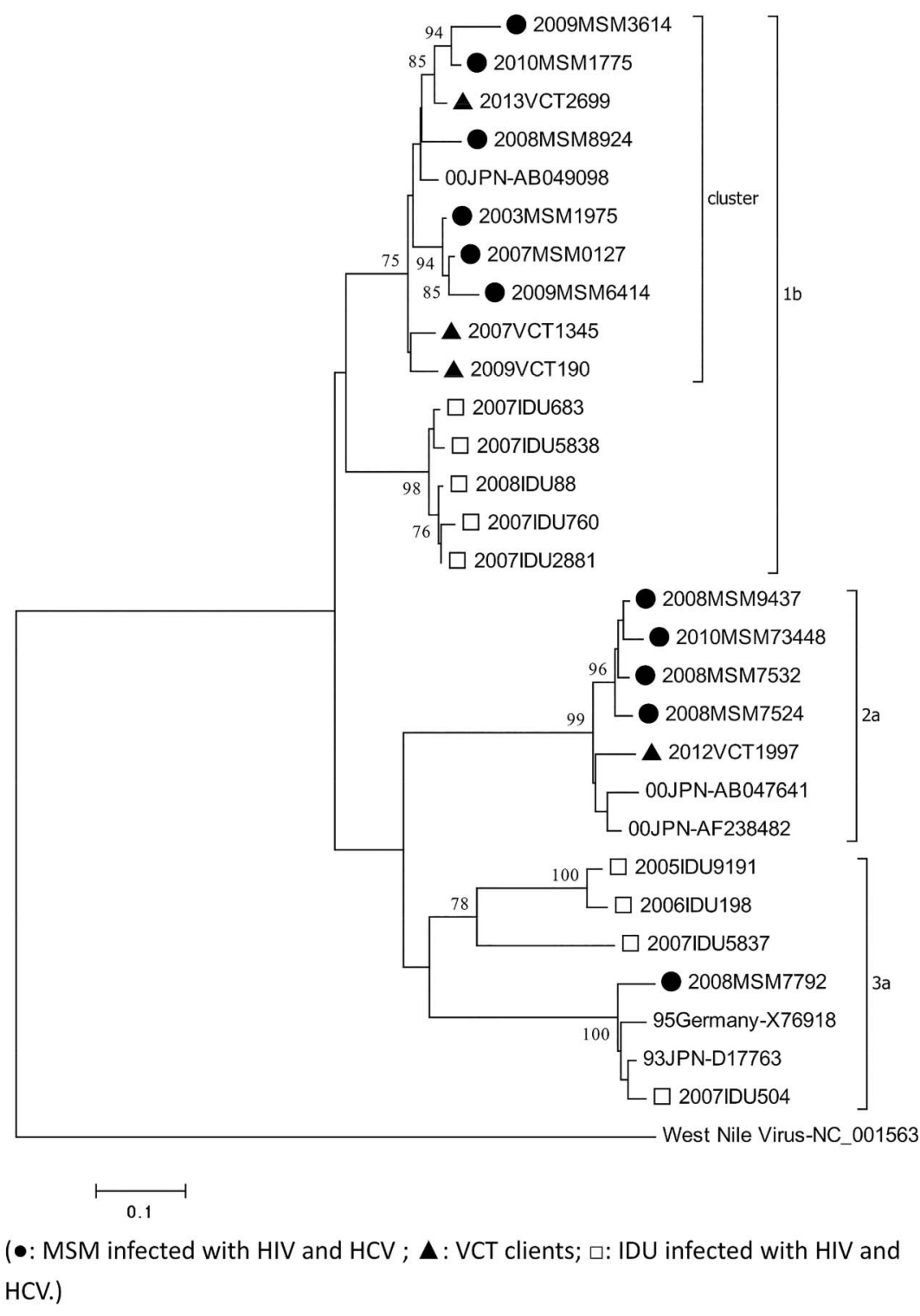

participation were associated with HCV acquisition in univariate analysis; moreover, sex in a group (more than two individuals) was the strongest predictor for HCV acquisition in this study. Similarly, Schmidt et a $t^{22}$ identified rectal trauma with bleeding, receptive fisting without gloves (or with shared gloves), group sex and nasally administered drugs were significant risks. The authors emphasised that rectal bleeding caused by fisting or prolonged anal intercourse leads to lesions in anal mucosa, which could serve as a portal of entry and facilitate HCV transmission. However, our questionnaire, in addition to querying on condom use, did not include inquiries about risk behaviours that may cause bleeding. ${ }^{25}$ Thus, we were not able to assess if those risky behaviours were associated with HCV seroconversion in our study.

There are several limitations in our study. First, we used a questionnaire to collect information on sexual risk behaviours. It is difficult to avoid recall bias of VCT clients and all data were self-reported; therefore we were not able to verify the information provided. Second, we did not perform HCV RNA for all clients who were HCV-seronegative. This could underestimate the incidence rate of $\mathrm{HCV}$ infection. Third, the number of HCV seroconversion cases remains small, which may preclude us from identifying other associated factors with HCV seroconversion. Fourth, our study was conducted at a single VCT site in Taiwan. More surveillance studies are warranted to confirm our findings of an increasing incidence rate of HCV infection in HIV-negative populations engaged in risk behaviours for STIs in Taiwan and other countries in the Asia-Pacific region.

In conclusion, we demonstrate an increasing trend of recent HCV infection among individuals who sought VCT services in Taiwan. Syphilis and having HIV-positive partners are two factors that are independently associated with recent HCV seroconversion.

Author affiliations

${ }^{1}$ Department of Internal Medicine, Tzu-Chi Hospital and Tzu-Chi University College of Medicine, Hualien, Taiwan 
${ }^{2}$ Departments of Internal Medicine, National Taiwan University Hospital and National Taiwan University College of Medicine, Taipei, Taiwan ${ }^{3}$ Departments of Laboratory Medicine, National Taiwan University Hospital and National Taiwan University College of Medicine, Taipei, Taiwan ${ }^{4}$ Department of Clinical Laboratory Sciences and Medical Biotechnology, National Taiwan University College of Medicine, Taipei, Taiwan ${ }^{5}$ Center of Infection Control, National Taiwan University Hospital, Taipei, Taiwan

Acknowledgements The authors would like to thank the VCT clients for participating in this study.

Contributors $\mathrm{C}-\mathrm{CH}$ contributed to the study conception and design. S-YC Y-CS and C-HW carried out the laboratory work. P-YW, Y-ZL and L-HC collected the demographic data. W-CL and J-CT performed the statistical analysis. J-CT, who interpreted the data and wrote the draft, was the lead author. C-CH, H-YS and S-CC supervised and revised the work critically for intellectual content. All the authors contributed to this manuscript.

Funding This work was supported by Taiwan Centers for Disease Control (grant number DOH102-DC-1401 and MOHW103-CDC-C-114-000405 to $\mathrm{C}-\mathrm{CH})$.

Competing interests None declared.

Patient consent Obtained.

Ethics approval This study was approved by the Research Ethic Committee of National Taiwan University Hospital.

Provenance and peer review Not commissioned; externally peer reviewed.

Data sharing statement No additional data are available.

Open Access This is an Open Access article distributed in accordance with the Creative Commons Attribution Non Commercial (CC BY-NC 4.0) license, which permits others to distribute, remix, adapt, build upon this work noncommercially, and license their derivative works on different terms, provided the original work is properly cited and the use is non-commercial. See: http:// creativecommons.org/licenses/by-nc/4.0/

\section{REFERENCES}

1. Bica I, McGovern B, Dhar R, et al. Increasing mortality due to end-stage liver disease in patients with human immunodeficiency virus infection. Clin Infect Dis 2001;32:492-7.

2. Operskalski EA, Kovacs A. HIV/HCV co-infection: pathogenesis, clinical complications, treatment, and new therapeutic technologies. Curr HIVIAIDS Rep 2011;8:12-22.

3. Sherman KE, Rouster SD, Chung RT, et al. Hepatitis C virus prevalence among patients infected with human immunodeficiency virus: a cross-sectional analysis of the US adult AIDS Clinical Trials Group. Clin Infect Dis 2002;34:831-7.

4. Alter MJ. Epidemiology of viral hepatitis and HIV co-infection. $J$ Hepatol 2006;44:S6-9.

5. Sun HY, Ko WC, Tsai JJ, et al. Seroprevalence of chronic hepatitis $B$ virus infection among Taiwanese human immunodeficiency virus type 1-positive persons in the era of nationwide hepatitis B vaccination. Am J Gastroenterol 2009;104:877-84.

6. Tseng YT, Sun HY, Chang SY, et al. Seroprevalence of hepatitis virus infection in men who have sex with men aged 18-40 years in Taiwan. J Formos Med Assoc 2012;111:431-8.

7. Lee HC, Ko NY, Lee NY, et al. Seroprevalence of viral hepatitis and sexually transmitted disease among adults with recently diagnosed HIV infection in Southern Taiwan, 2000-2005: upsurge in hepatitis $C$ virus infections among injection drug users. J Formos Med Assoc 2008;107:404-11

8. Sun HY, Cheng CY, Lee NY, et al. Seroprevalence of hepatitis B virus among adults at high risk for HIV transmission two decades after implementation of nationwide hepatitis $B$ virus vaccination program in Taiwan. PLoS One 2014;9:e90194.

9. Ghosn J, Pierre-Francois S, Thibault V, et al. Acute hepatitis C in HIV-infected men who have sex with men. HIV Med 2004;5:303-6.

10. Gotz HM, van Doornum G, Niesters HG, et al. A cluster of acute hepatitis $C$ virus infection among men who have sex with men- results from contact tracing and public health implications. AIDS 2005;19:969-74.

11. Gambotti L, Batisse D, Colin-de-Verdiere N, et al. Acute hepatitis $C$ infection in HIV positive men who have sex with men in Paris, France, 2001-2004. Euro Surveill 2005;10:115-17.

12. Serpaggi J, Chaix ML, Batisse D, et al. Sexually transmitted acute infection with a clustered genotype 4 hepatitis $C$ virus in HIV-1-infected men and inefficacy of early antiviral therapy. AIDS 2006;20:233-40.

13. van de Laar TJ, van der $\mathrm{Bij} A K$, Prins $\mathrm{M}$, et al. Increase in HCV incidence among men who have sex with men in Amsterdam most likely caused by sexual transmission. J Infect Dis 2007;196:230-8.

14. Ghosn J, Deveau C, Goujard C, et al. Increase in hepatitis C virus incidence in HIV-1-infected patients followed up since primary infection. Sex Transm Infect 2006;82:458-60.

15. Richardson D, Fisher M, Sabin CA. Sexual transmission of hepatitis $\mathrm{C}$ in MSM may not be confined to those with HIV infection. J Infect Dis 2008;197:1213-14, author reply 14-15.

16. van der Helm JJ, Prins $M$, del Amo J, et al. The hepatitis $C$ epidemic among HIV-positive MSM: incidence estimates from 1990 to 2007. AIDS 2011;25:1083-91.

17. Giraudon I, Ruf $\mathrm{M}$, Maguire $\mathrm{H}$, et al. Increase in diagnosed newly acquired hepatitis $\mathrm{C}$ in HIV-positive men who have sex with men across London and Brighton, 2002-2006: is this an outbreak? Sex Transm Infect 2008;84:111-15.

18. Sun HY, Chang SY, Yang ZY, et al. Recent hepatitis $C$ virus infections in HIV-infected patients in Taiwan: incidence and risk factors. J Clin Microbiol 2012;50:781-7.

19. Rauch A, Rickenbach $M$, Weber R, et al. Unsafe sex and increased incidence of hepatitis $C$ virus infection among HIV-infected men who have sex with men: the Swiss HIV Cohort Study. Clin Infect Dis 2005;41:395-402.

20. Urbanus AT, van de Laar TJ, Stolte IG, et al. Hepatitis C virus infections among HIV-infected men who have sex with men: an expanding epidemic. AIDS 2009;23:F1-7.

21. Witt MD, Seaberg EC, Darilay A, et al. Incident hepatitis $C$ virus infection in men who have sex with men: a prospective cohort analysis, 1984-2011. Clin Infect Dis 2013;57:77-84.

22. Schmidt AJ, Rockstroh JK, Vogel M, et al. Trouble with bleeding: risk factors for acute hepatitis $\mathrm{C}$ among HIV-positive gay men from Germany-a case-control study. PLOS ONE 2011;6:e17781.

23. Scott C, Day S, Low $\mathrm{E}$, et al. Unselected hepatitis $\mathrm{C}$ screening of men who have sex with men attending sexual health clinics. $J$ Infect 2010;60:351-3.

24. Su YC, Liu WC, Chang LH, et al. Incidence of recent HCV infection among persons seeking voluntary counselling and testing for HIV and sexually transmitted infections in Taiwan. J Int AIDS Soc 2014:17:19640.

25. Wu H, Wu PY, Li SY, et al. Maximising the potential of voluntary counselling and testing for HIV: sexually transmitted infections and HIV epidemiology in a population testing for HIV and its implications for practice. Sex Transm Infect 2012;88:612-16.

26. Chang SY, Yang CL, Ko WS, et al. Molecular epidemiology of hepatitis $D$ virus infection among injecting drug users with and without human immunodeficiency virus infection in Taiwan. J Clin Microbiol 2011;49:1083-9.

27. Kumar S, Tamura K, Nei M. MEGA3: Integrated software for Molecular Evolutionary Genetics Analysis and sequence alignment. Brief Bioinform 2004;5:150-63.

28. Nishijima $\mathrm{T}$, Shimbo $\mathrm{T}$, Komatsu $\mathrm{H}$, et al. Incidence and risk factors for incident hepatitis $\mathrm{C}$ infection among men who have sex with men with HIV-1 infection in a large urban HIV clinic in Tokyo. J Acquir Immune Defic Syndr 2014;65:213-17.

29. Lin AW, Wong KH, Chan K. More safer sex intervention needed for HIV-positive MSM with higher education level for prevention of sexually transmitted hepatitis C. J Int AIDS Soc 2014;17:19663.

30. Sherman KE, Shire NJ, Rouster SD, et al. Viral kinetics in hepatitis $\mathrm{C}$ or hepatitis $\mathrm{C} /$ human immunodeficiency virus-infected patients. Gastroenterology 2005;128:313-27.

31. Briat A, Dulioust E, Galimand J, et al. Hepatitis C virus in the semen of men coinfected with HIV-1: prevalence and origin. AIDS 2005;19:1827-35.

32. Lattimore S, Thornton A, Delpech V, et al. Changing patterns of sexual risk behavior among London gay men: 1998-2008. Sex Transm Dis 2011;38:221-9.

33. Danta M, Brown D, Bhagani S, et al. Recent epidemic of acute hepatitis $C$ virus in HIV-positive men who have sex with men linked to high-risk sexual behaviours. AIDS 2007;21:983-91. 